\title{
Developing Strategies in System Level Model of Smart Cyber Physical System
}

\section{László Horváth}

Óbuda University, Doctoral School of Applied Informatics and Applied Mathematics and John von Neumann Faculty of Informatics, Bécsi u. 96/b, H1034 Budapest, Hungary, horvath.laszlo@nik.uni-obuda.hu

\begin{abstract}
It was long way to current highly integrated and smart modeling and simulation in engineering. This way started with separated problem solving for representation, manufacturing control, and visualization of mathematical surfaces. By now, autonomous engineering model system (EMS) serves lifecycle innovation and engineering of systems operated autonomous intelligent industrial and commercial products. Modeling platforms constitute one of the most important and extensive application areas in information technology. Advanced EMS is highly integrated, multidisciplinary, and multipurposed model structure. Any contribution to EMS must use contextual object modeling (CM) and continuous engineering (CE) which are within the most important achievements in engineering during the past two decades. Considering the above situation, this paper introduces the novel organized content model (OCM) approach, concept, and methodology as contribution to integrated and lifecycle serving model system of highly automated products. OCM organizes driving contexts for lifecycle of modeled product. It is an extension to the currently prevailing EMS structure to contribute fulfilling the new requirement of active context based integration of virtual (EMS) with physically operating cyber physical system (CPS) products. Other essential requirement is that relevant objects in EMS and CPS must be contextually driven by higher level decisions, intellectual property (IP) items, and human intervention attempts while EMS and CPS are increasingly autonomous. Therefore, main purpose of OCM is to support active driving contextual connections for EMS and CPS. In this way, this paper discusses integrated model of highly automated complex product system, extended OCM for situation controlled product system, and Support of Situation Awareness by OCM. Research for OCM is done at the Laboratory of Intelligent Engineering Systems (IESL). In this context, preliminary research at IESL and concept of configuring professional cloud organized modeling platform for the purpose of virtual research laboratory (VRL) are also included in this paper.
\end{abstract}

Keywords: smart cyber physical system; engineering model system; system level modeling; situation based modeling; organized content model 


\section{Introduction}

This paper introduces latest methodological and communication contributions to system level engineering modeling. Work for these contributions was motivated by more than two decades development of integrated model systems to represent aircraft, car, and other very complex, strongly multidisciplinary, systems operated, and situation controlled industrial product structures in autonomous engineering model system (EMS). The internationally recognized Laboratory of Intelligent Engineering Systems (IESL) joined to research in very extended and comprehensive virtual technology for EMS. The main objective was to achieve integrable principles, methods, and structures.

Due to its fantastic multidisciplinary development including recently emerged new essential modeling issues, the latest engineering technology is a challenge to understand for experts with classical modeling knowledge and experience. Autonomous operation of EMS requires consistent structure of knowledge based driving contextual connections, which includes all object parameters. Modeling related meaning of the word "driving" may be unusual for readers of this paper. Meaning here is setting value of an object parameter by value of related parameters in EMS objects using contextual connection definitions established between the relevant parameters. It must be also noted that word "definition" is used for a human or systems activity which inserts objects and their parameters with actual values in EMS.

Advanced EMS includes generic, self-adaptive, theory-experience integrated, active knowledge driven, systems representation enabled and, autonomous decision capable component models. Accommodating huge amount of new and changed contexts is unavoidable during the integrated innovation and life cycle of a represented product. Variants, developments, and improvements of product must be handled using the same EMS during lifecycle. By now, product structure is often composed by several millions of systems organized components. Shifting industrial engineering to much higher level enforced integrating fundamental, problem solving purposed, and industrial and commercial product related research activities into EMS. Considering so many new terms and new interpretation of old terms, author of this paper apologizes if the reader cannot find explanation for all of them. References in this paper and references in the cited papers include all necessary explanations.

Product model was a main concept in the ISO supported integrated product information model (IPIM) during the 90s. Product model system is called as EMS in this paper. It is consistent representation of generic product system which recently includes cyber units controlled physical units and sensor networks. In this context, term "product system" refers to product, which is modeled as system or system of systems. EMS must be capable of behaving as planned or analyzed product. EMS level model structure was first applied in model based product 
lifecycle management (PLM). Structure of a system level EMS requires levels for the extension of classical physical level product model to representation of systems. Driving an EMS is the capability to operate consistent structure of active outside and inside contexts. Situation-controlled product system is developed to achieve its autonomous features. Cyber.units are capable to recognize situation, decide physical actions in accordance with recognized situation then real time control those physical actions. Situation awareness is an essential new capability at systems and intervening humans.

IESL has been active in the organization of Óbuda University for fifteen years. It was established in year 2005 [1] to support university research and graduate higher education in theory, methodology and systemics of engineering modeling. Research in EMS was intended as main area considering integrated application of mathematics, physics, systems engineering, intelligent informatics, and area specific industrial object modeling. Recently, IESL is undergone a laboratory development which aims availability of research enabled systems level engineering modeling platform (EMP) capabilities to introduce experimental model system based research in wide context. In this level, EMP is very complex and provides modeling and other needed organizational capabilities for lifecycle engineering of system operated product using EMS. Availability of suitable engineering modeling platform makes establishing the Virtual Research Laboratory (VRL) at the Doctoral School of Applied Informatics and Applied Mathematics (DSAIAM) possible. Main concept of VRL is experimental model centered research [3] mainly for doctoral research serving Ph.D. projects. VRL is main development of IESL.

This paper introduces results from research in organized content model (OCM) which fills the gap between EMS and driving contexts which connect EMS with world outside of it. OCM includes, among others, representations for decision making, human intervention, knowledge, and former experience. Several former OCM structure variants were proposed among others for driving of systems enabled [4] and cyber physical system (CPS, [36]) connected [5] EMS during the past decade. Main issues in this paper are integrated model of highly automated complex product system, analysis of contexts in OCM, extended OCM for situation controlled product system, and support of situation awareness by OCM. Research for OCM is done at the IESL. Preliminary research at IESL and concept of configuring professional cloud organized EMP for the purpose of virtual research laboratory (VRL) are also included in this paper.

Although the author of this paper tried writing with professional comprehensibility, the results presented here inherently require relatively depth knowledge about latest engineering modeling achievements as well as leading engineering platforms applied at implementation of these achievements in leading practice. 


\section{Preliminary Research}

Research in knowledge driven definition, generation, and application of engineering models to enhance development, production, and application of industrial and commercial products is long-term program at the IESL and its predecessor laboratories from the 90s. This program systematically renewed several times to follow new milestones during the above period. To achieve this, the modeling software system was regularly upgraded to represent the latest theories, methodologies, and systemics. This sector of paper is a selection of former contributions which have effect on current and future research at the IESL [11-32].

In the second half of the 90s, one of the main issues was modeling of process objects in connection with product component features. Generic process features were defined and represented by Petri net in [11] together with built-in knowledge for the evaluation of Petri net. Evaluation procedure defined actual status of Petri net to achieve a process instance. This method was applied at manufacturing process. As contribution to solutions in model integration, a methodology was proposed to attach designer intent knowledge to form feature representation, among others to improve communication between product designer and production engineer [12]. A method was introduced in [13] to enhance integration of process modeling with simultaneous and collaborative engineering methodology. Actual issues in intelligent computing were studied regarding computer assisted engineering activities in [14]. Research results in associative connections for models of mechanical parts were published in [15] and in integration of expert knowledge in behavioral feature enabled product model in [16].

Importance of product behavior and its human intent origin were recognized, and relevant modeling method was proposed in [17]. Book [18] introduced and organized model based problem-solving methods in representative areas of engineering. Paper [19] discussed a new model representation of human intent to achieve capability of product model objects for self-modification in accordance with environmental changes.

When intelligent computing supported decision-assistance had become a main concern in model-based engineering, related issues were analyzed in [20]. A new approach to handle product changes in product model and a method for the related processing in product model assisted engineering were introduced in [21]. Content which represents knowledge for driving the generation of model information is proposed in [22] as one of the main initiatives during the long-term research. Developing strategies in engineering placed new emphasis on model representation of human intent at the beginning of the new century. Joining to this trend, human intent representation was proposed for product model with the emphasis on the intensive knowledge processing capabilities [23]. In this context, 
knowledge technology methods were proposed for application in industrial product modeling [24], for integration the aspect of engineering process in virtual product definition [25], and for adaptive knowledge driving at definition of product objects [26].

Recently, research for OCM at the IESL has changed for issues in driving of object parameters in case of model systems serving engineering of highly automated and increasingly autonomous industrial products. This changed situation needed concept for system level OCM. Considering current industrial modeling practice, driving object parameters in requirements, functional, logical, and physical (RFLP) structured EMS was decided [8]. The RFLP structure is IEEE standardized and it is one of the essentials in systems engineering (SE). It integrates SE methodology with the former physical level product modeling. Flexibility and reliability of an OCM installation is highly depends on consistent structure of contextual connections [6]. Anyway, contextual modeling has outstanding importance in recent achievements in engineering modeling [38].

By the second decade of our new century, modeling of situation controlled, and systems operated product became key issue. Hundreds of systems can cooperate among others in cars, aircrafts, robot based assembly systems, and hardwaresoftware systems. Analysis of possibilities to apply active knowledge for situation based driving at definition of product motivated the development of new situation driven definition processes and entities in OCM [27]. Paper [28] introduced the initiative, behavior, context and action structured driving content representation and its application at control of component generation in RFLP structure.

Multilevel abstraction was applied at definition method for self adaptive model and relevant model structure [29] as contribution to higher abstraction based calculation of component parameters in RFLP structure. Organic integration of the proposed model structure into PLM model structure was considered.

Paper [30] discusses the need to improve the formerly proposed initiative, behavior, context, and action content structure, proposes extended and restructured version of this structure, and introduces new approach to driving parameters in contextual product model components. A methodology for intelligent driving of parameters in functional and logical components of RFLP structured EMS in system level engineering is introduced in [31]. Formerly published concept of virtual engineering space (VES) is applied at development of new method for the generation of self-adaptive generic model objects using contextual initiatives for knowledge content background in [32]. 


\section{Integrated Model of Highly Automated Complex Product System}

Current industrial practice requires highly integrated autonomous EMS to support development, production, and application activities at engineering of very complex and highly automated products. This EMS is also very complex, and it is developed and applied in product lifecycle organized integrated engineering process. Huge number of product objects and object parameter contexts are defined and represented in highly integrated, generic, and autonomous EMS. Representations, algorithms, decision support procedures, and other advanced computing related entities are applied at definition of consistent context structure in EMS. Beyond contexts between inside object parameters, EMS as product model system (PMS) is driven by outside contexts for human intervention, higher level decisions, former results, and intellectual property. Therefore, relevant EMS object parameters are in contextual connection with outside driving object parameters. Finally, relevant PMS object parameters are in contextual connection with field operating product and production system.

Situation-controlled and systems-organized autonomous product includes extended cyber units which are authorized for real time control of physical units. PMS is continuously changed during lifecycle of product. Conventional sense of product does not apply any more in advanced industry. Contextual connection between PMS and cyber units of CPS has increasing role. For analysis of the above complex system, model of extended product system (EPS) was conceptualized (Fig. 1). In EPS, PMS is the virtual form of product and interacts with physical product for mutual update, upgrade, intelligent cooperation, and intelligent control. Consistency of context structure must be assured in the full EPS during its lifecycle. As it can be seen in Fig 1, EPS receives outside driving contexts in five groups. These groups are participant interventions, higher level decisions, laws and standards, intellectual property, and authorized operator actions. Development of PMS needs application of powerful contextual modeling methodology, which is considered as one of the most important recent achievents in virtual engineering. In the EPS scenario, role of OCM is coordinated diving of PMS and cyber units of CPS with the possibility of bypassing. As it can be seen in Fig. 1, the driving connection is with the situation recognition unit of CPS cyber sector referring to the unavoidable situation recognition and its OCM support in the future. Recent methodology of continuous engineering (CE, [6]) has critical role at lifecycle development of EPS.

Interactions for context accommodation in OCM or/and PMS include procedures to receive, check, and accommodate contexts for EPS. This is one of the two main contributions in this paper (see the next section). The other main contribution is the organized content model (OCM) itself. OCM fills the gap between context accommodation interactions and RFLP structured PMS as well as situation 
recognition. As it can be seen in Fig. 1, authorized engineer can decide bypass of OCM at product which does not require representation of complex context structure. The remaining of Fig. 1 shows relevant functional units in cyber units of a CPS product. Situation recognition unit is responsible for situation awareness of highly automated product with autonomous functions. Extended OCM is proposed in section 5 of this paper to assist situation awareness both in approved systems and at authorized humans on duty. Physical activities are decided by decision unit and executed by relevant control in accordance with commands deployed by execution unit.

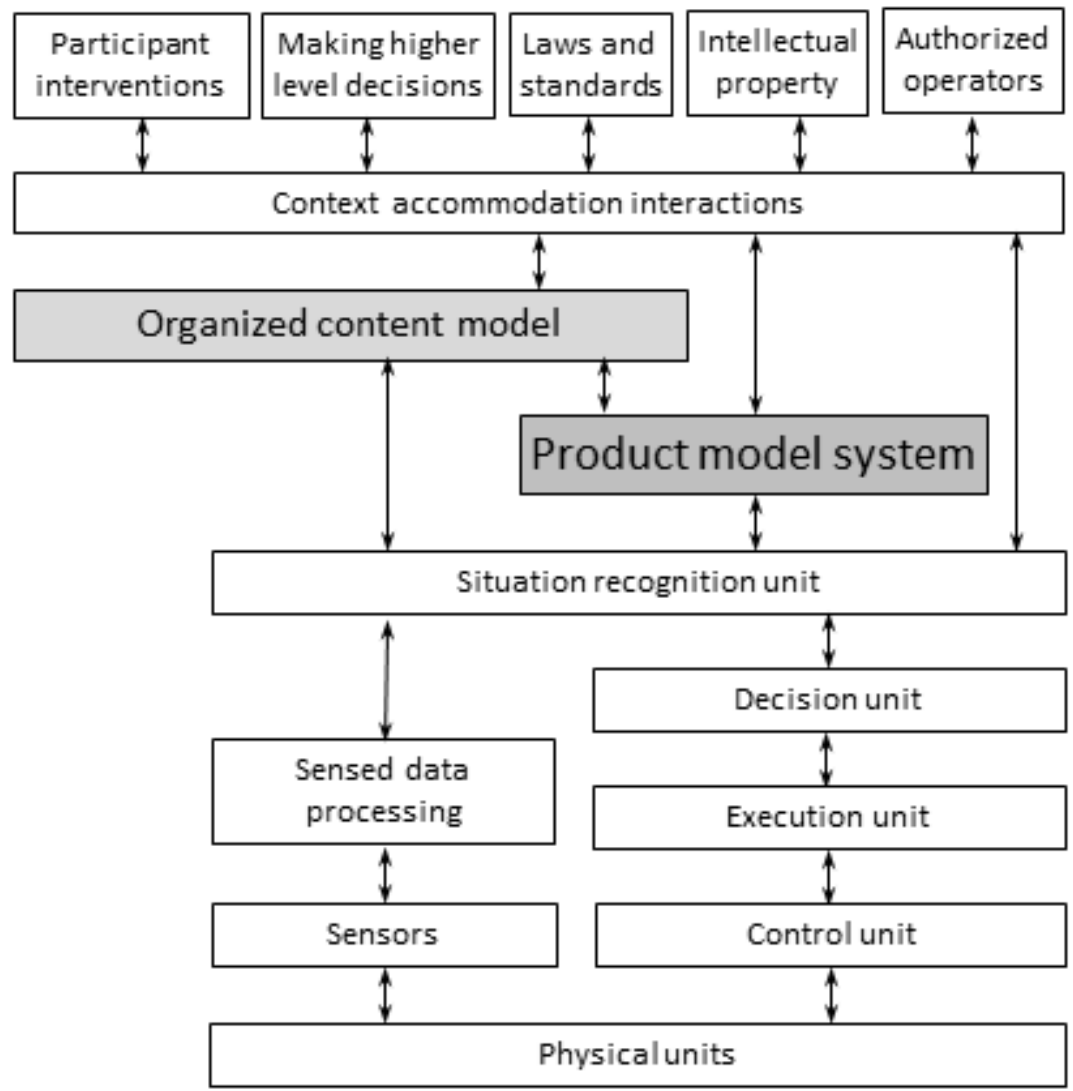

Figure 1

Model of the extended product system (EPS)

In the past, classical product was operated by chains of mechanical connections with power drives at given points. By the development of mechatronics, electronic units were included to control electrical so that indirectly mechanical units. The next milestone was the computer control which step-by-step integrated the formerly individual controls. This trend has led to systems organized product. 
In development of a systems-organized product, first the systems are defined on the levels of functions and logical connections. It is obvious that systems operated product is multidisciplinary [37]. In a highly automated product, cyber units control existing physical units and accommodate control of newly announced physical units during the life cycle of product. Sensors carry actual physical operation parameter values in accordance with inquiries from cyber units. Cyber units flexibly reconfigured and modified during the life cycle of product.

RFLP structured PMS is defined for systems operated generic industrial or commercial product or product family and it includes functional (F) and logical (L) structures of product components to establish and virtually execute behavior driven concept model of product [7]. Each component includes appropriate behavior representation to allow virtual execution of the above conceptual product model. This model includes contexts for the generation of physical component models as feature structures. In RFLP structured model, requirements (R) level elements drive functional (F) level components, F level components drive logical (L) level components, finally $\mathrm{L}$ level components drive physical (L) level components in a contextual structure.

Beyond improved capability of OCM to assist cyber units of CPS product, these cyber units are capable to carry actual information and even processed experience about physical object and process parameters to OCM and/or RFLP structured PMS. Continuous engineering (CE) processes are capable of real time consequence evaluation at any model or software modification. The above benefits motivated integration of OCM, RFLP structured PMS, and cyber units of CPS products in EPS (Fig. 1). This integration improves optimal as well as secure physical activities by the assistance of CE procedures.

\section{Contexts in OCM}

One of the main purposes of OCM is to assure consistency of actual active context structure for EPS. This is essential for the capability of continuous development, which is realized in physically operating product. When development of a product requires any change of cyber or physical units, OCM must communicate considering the changed configuration. Recognizing this new style of product development motivated the EPS concept and the related analyses which are reported in this paper below. It must be noted that OCM related key words such as information, content, and contexts are applied with diverse meanings in other technical areas.

Before further characterizing the role of OCM in EPS, some words must be devoted to the content, which is organized and represented in OCM. Representation of systems constituting PMS (EMS) requires higher abstraction in 
content for OCM than conventional physical level representation. This recognition was published thirteen years ago in [9] where content was defined as information content acting as background of model information for information level product model. As it was explained above in this paper, content item represents or generates driving context.

Although context item may come from various sources, its origin is always human brain as in case of any information. Connection chain between original source and the context item, which is communicated with EPS generally includes transformations. Russell Ackoff classified human brain content into data, information, knowledge, understanding, and wisdom categories [10]. In this categorization, data is content which does not have any meaning. Information is content which has meaning by relational connections of data. Information answers questions like who, what, where, and when. In this way, data with contextual connection is considered as information. Information content in OCM is required and suitable to drive relevant target parameters in relevant target object.

However, information does not answer two main OCM item related questions that how and why. Ackoff proposed knowledge to answer how while understanding to answer why. In this concept, understanding is in interposition between knowledge and wisdom. Although wisdom is not an issue in this paper, its representation probably will be increasingly important for future autonomous products in the industry so that for future OCM. Considering the current product development trend, high-level automation related concepts will be essential for everyday engineering in the future.

It can be concluded from the above that one of the main purposes of OCM development must be to provide answer the questions how and why. Driving of PMS and cyber unit object parameters by actual OCM content must be analyzed in this level. It is obvious that OCM item represents active driving knowledge. The next question is how can understanding be represented as OCM content. This question will be answered by future research at the IESL.

Main steps of context item processing in OCM for driving relevant parameters of RFLP structure objects can be followed in Fig. 2. As it can be seen in Fig. 1, OCM receives contexts from the context accommodation interactions. This context is passed to RFLP structured product model object parameter(s) after suitable processing. At conventional human or formal object parameter connection based definition of objects in RFLP structure, this connection is direct. This is denoted in Fig. 2 by dashed line. Definition of RFLP structure objects using many contexts and tracking the changes of these contexts during life cycle of product are practically would become impossible in direct way considering high product complexity as in case of CPS system. Fig. 2 shows a proposed process which can replace manual or other direct definition of contextual objects of RFLP structure.

The context accommodation interactions function passes any eligible context to the space of received driving contexts. Eligibility of a context item to pass into the 
OCM is checked using relevant protocol, which is effective in the actual engineering environment. OCM related protocols are not issues in this paper and are subjects of future research at IESL. Definition of contexts for OCM is transformation to make contexts suitable for the definition or extension of relevant OCM substructure element(s). Structure of OCM is explained in the next section of this paper. New context in an OCM element become active and new or modified contextual connection with other related elements is activated in coordination with existing contexts along contextual chains of object parameters. In this way, any new or modified context is coordinated with all related active contexts and actual coordinated contexts drive related object parameters in RFLP structure as driving context.

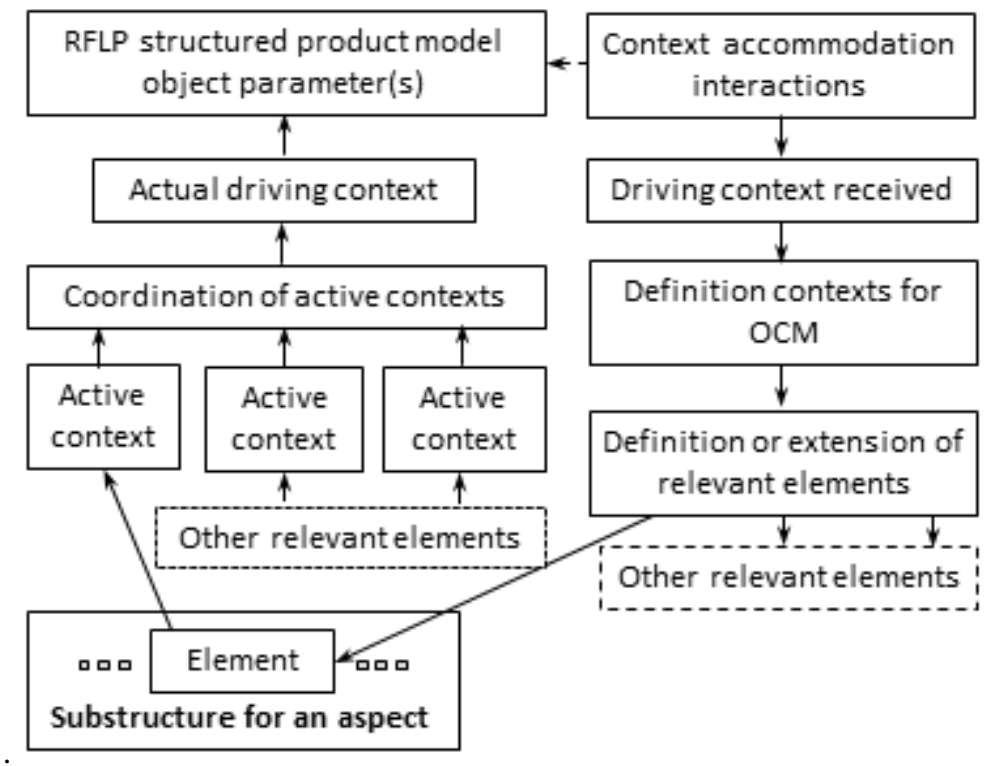

Figure 2

Processing contexts in OCM for RFLP structure

Eligibility check must be included to assure suitability and security of context item received for the accommodation in OCM. Main steps of the proposed eligibility check are explained in Fig. 3. After receiving, driving context is undergone a procedure to recognize the relevant aspects then elements to be originally defined or completed within each aspect in OCM. Aspects are represented in substructures of OCM levels. This step includes checking the new context in accordance with protocol in effective. As a result of the above procedure, any obviously erroneous context is refused. Because new contexts enrich important content during continuous development of EMS, it is essential not to trash any valuable context. In argued case, it is advised to allow online context correction for trusted sources. Status of context is recorded, and 
appropriate notice is generated for the owner of the context. It must be recognized that communication with autonomous sources must be increasingly considered in the future.

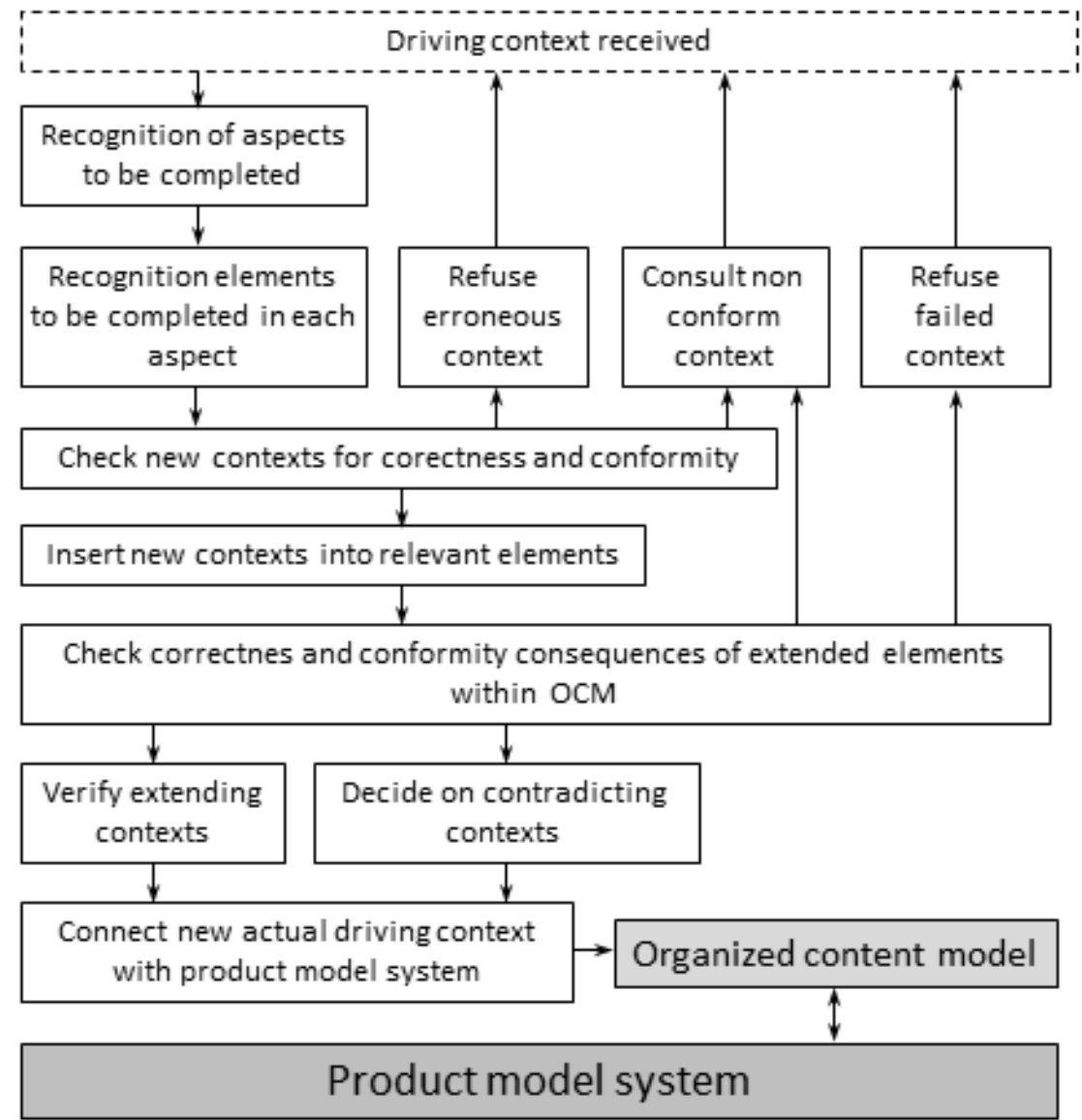

Figure 3

Essential accommodation of contexts in OCM

When it is placed in the appropriate elements within the relevant aspect(s), any new context is checked for correctness and conformity. Correctness is checked using a checklist provided by the effective protocol. Protocol includes a set of firm contexts to be fulfilled. Conformity is more complicated than correctness because it can depend on any existing context with which the checked context is connected or interdependent. Any obviously not correct context is refused with notice sent to its owner. Non conform context is discussed by OCM responsible and owner humans or system. In case of disagreement, checked context is passed to higher level decision making or refused in accordance with the effective protocol. Anyway, discussion is required except for obviously correct and conform cases. 
After successful check of correctness and conformity, insert of context in relevant elements of OCM is finalized. The next step is checking correctness and conformity consequences on extended elements within OCM along relevant active context chains. Appropriate extending contexts are verified while additional decisions are required on contradicting contexts. In final decision, previously accepted contexts in context chain are often need modification or even refuse. A finally approved new actual context in OCM is actively connected in EMS with permission for its driving effect.

The above procedure may seem as very complicated together with strict development of hard to survey OCM structure. Really, the recent practice in EPS has become very complex and different from previous practice. OCM which is proposed in the next section of this paper is an attempt to organize so many different contributions from so many different sources into consistent driving structure to facilitate decisions which aware of all essential as well as valuable contributions. This is a contribution to new level of engineering owing to the capabilities of contextual autonomous engineering modeling. OCM is intended as a possible contribution to solve recently typical problems caused by locally correct results which are failed at its integration in the demanded much wider context.

\section{Extended OCM for Situation Controlled Product System}

A new OCM model structure was conceptualized to be eligible to provide context support for RFLP structured PMS and cyber units of physically operating product. Formally, OCM consists of levels and includes sequence of substructures on each level. Each substructure serves an aspect of content and consists of dynamic structure of port connected blocks. A block represents an element of substructure. Levels and substructures within a level are placed in mandatory contextual chains. Each level serves closely connected group of aspects. Any contextual element can be connected by additional context if the new context does not break any effective context. Context item is represented in OCM and it is placed in one or more element in one or more substructures. Structure of OCM is planned to realize using functionality, which is available for RFLP structure. Life cycle development of OCM is a great challenge for engineers and it is one of the highest-level engineering activities in product engineering.

Publications about former relevant OCM variants which are specially designed for driving of systems enabled [4] and CPS connected [5] PMSs introduce basic theory, methodology, and systemics of OCM. This sector of paper is restricted to the new OCM structure variant which extends the above formerly developed versions to drive PMS which represents situation-controlled product. 
A specific feature of the proposed OCM structure is placing new emphasis on situation awareness. Situation awareness is equally important at smart system which applies it at its autonomous activities and at human who is on duty at operating this system. Human on duty should be available to correct a failed autonomous decision at any time. Self-awareness, self-adaptation, and selfoptimization were emphasized as main interacting capabilities for situation awareness in [33]. In the concept of [33], self-awareness recognizes situation using behavior, self-adaptation modifies objects acting on situation, and selfoptimization improves quality of decisions on adaptive modifications.

The proposed OCM consists of five levels in a contextual chain (Fig. 4). This contextual chain is enforced by procedures at development and application of OCM for saving integrity and consistency of OCM. This also applies to the sequence of substructures within each level. In the proposed OCM, levels are defined to contextually organize aspects for model construction, product behavior, systems which operate the product, control of automatic equipment and devices, and human-system connections. Elements in sublevels are in driving contextual connection with sources from which contexts are accommodated for driving object parameters in RFLP structured EMS, for human inspectors who are on duty at operating of product, and for connected cyber units of CPS system. Aspects and related content on levels are characterized below.

Construction aspects organize content for the construction of PMS. While required function and component contexts of product and the related process and procedure contexts are in a contextual sequence, outside contexts also drive elements in all these substructures.

Behavioral aspects have critical position in smart systems because virtual execution on system level of PMS relies upon behavior representation in components within RFLP structured PMS. On the other side, behavior is defined in the context of circumstances for situation. This level is an attempt to coordinated driving by these three groups of objects. It is critical at real time operation of systems within smart product structures. Behaviors are among main areas of future research at IESL.

Level Systems aspects is contribution to represent driving contexts for model of systems in RFLP structure. Current RFLP structures inherently represent systems implicitly on their functional and logical levels. Level Systems aspects organizes system related driving contexts in substructures for cooperating component systems. Units of a component system are defined for a given system, but they are organized in common substructure to represent their contexts mainly for their interaction. Finally, aspect of system operation is defined for system functions and related operational parameters. 


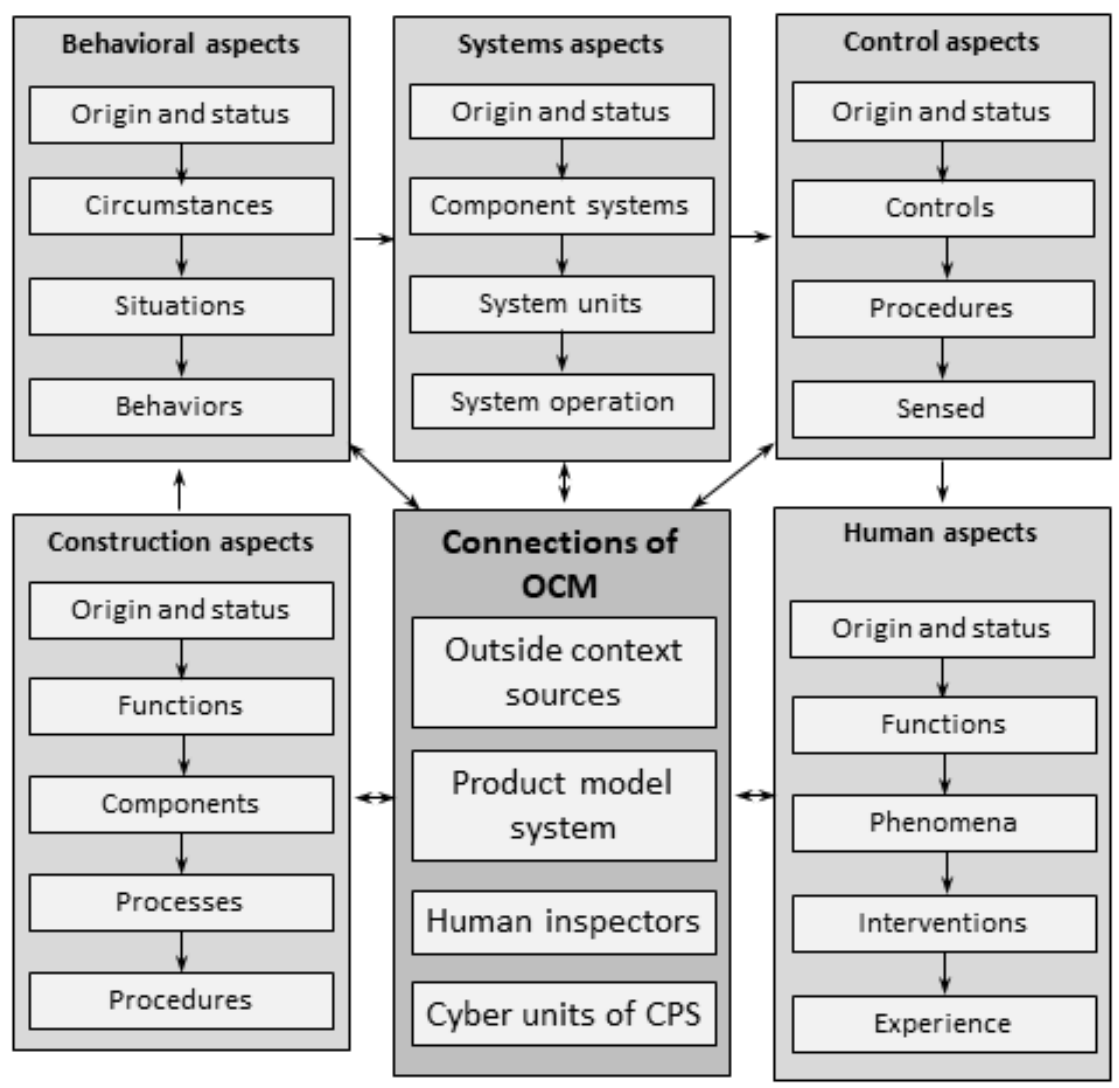

Figure 4

Main structure of the extended OCM

Level Control aspects is attempted to organize contexts for control equipment and devices. Activities of these objects are coordinated by cyber units in smart CPS product. Control equipment and associated devices are represented in PMS. While substructure Controls organizes driving contexts for control equipment and devices, driving contexts are also organized for procedures in PMS and CPS cyber units, and for sensed parameters which are real time processed in cyber units.

Finally, level Human aspects is devoted as contribution to solutions for the problem of human situation awareness with special attention to system fails at situation recognition when human on duty must correct the recognized situation to improve systems operation and prevent malfunction or even danger. Functions of product which are executed according to valid decisions in smart products are structured then phenomena contexts are organized in the context of functions. The next sub-level organizes possible interventions at system related functions. Finally experience organizes content items from past cases in the context of phenomena through relevant interventions. 
OCM is model level environment to achieve organized driving content for the other units in EPS. In this way, OCM is integrated to EMS so that in EPS. For that reason, implementation of OCM must be done in appropriately conditioned professional engineering modeling platform as it will be discussed in sector 7 of this paper. As in case of smart engineering [34] and smart CPS products, engineering with autonomous OCM requires participants who are in possession of all knowledge and experience needed by system level, multidisciplinary, large structure related engineering modeling activities.

\section{Support of Situation Awareness by OCM}

By now, situation awareness is one of main concerns in highly automated situation-controlled product systems especially when autonomous functions are supported. OCM related discussion of situation awareness needs answer for three essential questions. The first one is that how connection between autonomous PMS and CPS cyber sector can be enhanced by appropriately configured autonomous OCM. The second one is that how situation extension of OCM assists development and operation of autonomous product characteristics. The third one is that how to automate the process in Fig. 3 to cope with demands posed by autonomous features of product. Answers to these questions need research which must be extended to whole EPS (Fig. 1). Considering the above questions, OCM support of situation awareness needs suitably configured sets of contextual connections within OCM, between OCM and PMS, and between OCM and cyber units of product.

Three aspect chains with three aspects (substructures) in each, play important role at support of situation awareness by OCM (Fig. 5). Elements in Circumstances aspect represent content for correct recognition of situation and can be improved by situation-based experiences in CPS. Actual behavior can be correctly represented in this way. Level Control aspects provides equipment and device control drives together with the related procedures to enhance control by cyber units. In the other direction, sensed parameter values contribute to field improved driving content for models and procedures. Level Human aspects supports situation recognition by phenomena content, which is the context for required human intervention and, in this chain, enriches content with past proven and failed experience.

Process of situation recognition support by OCM can be followed in Fig. 5. Relevant cyber units provide decision chain for controllable physical units in CPS. This chain includes cyber functions for execution of physical actions and for deploy decided and prepared control on physical units (Fig. 5). In the meantime, sensor information about physical unit parameters is collected and processed. 


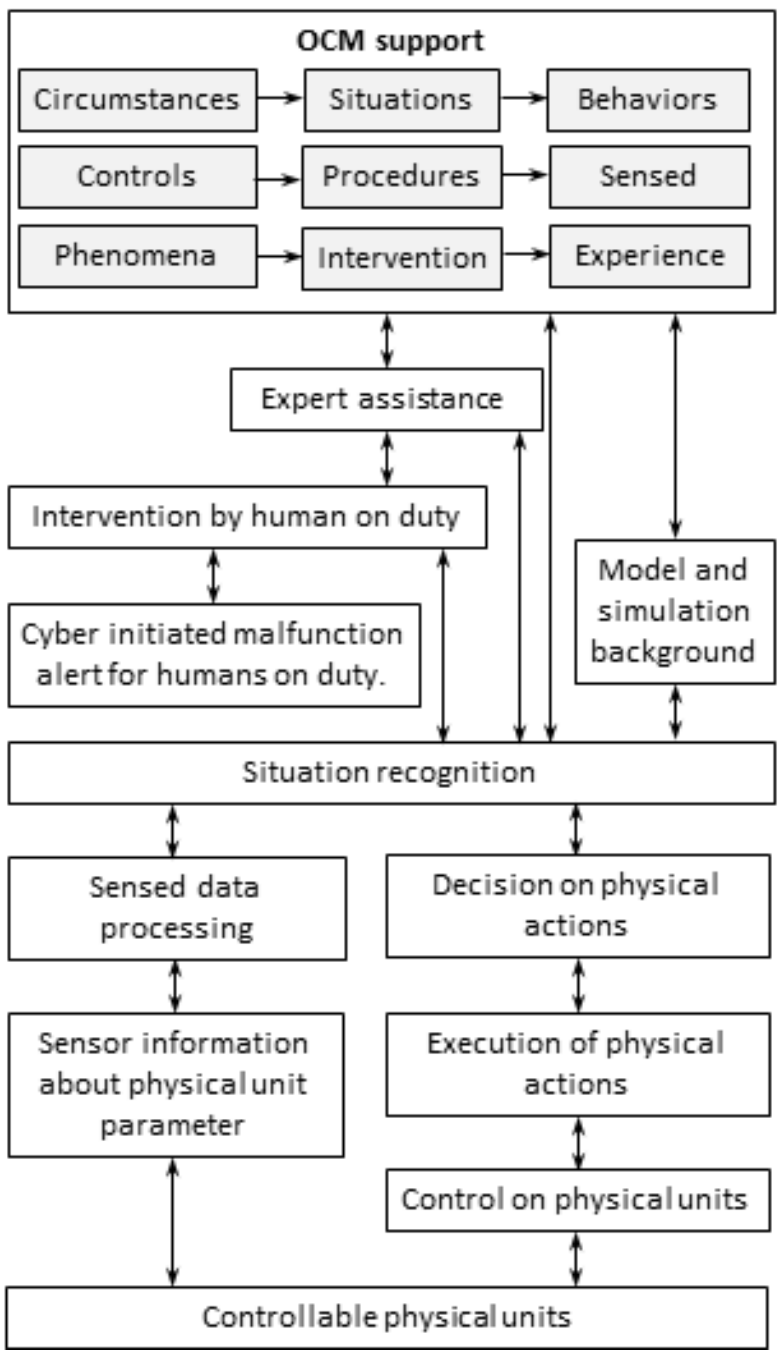

Figure 5

OCM support of situation awareness

In the schema in Fig. 5, responsibility of human on duty is continuous for intervention in case of CPS cyber initiated malfunction alert. This alert can be communicated with OCM directly, or through expert assistance. OCM is capable of expert assistance using content on the level Human aspects. Engineer on duty can be assisted by information about former similar cases with of without expert assistance. Any autonomous and human intervention is done through situation recognition. The challenge here is not only recognition and correction of situation but also the enforced immediate intervention. Therefore, the autonomous response of OCM is very important. 


\section{Implementation Issues}

Current high-level model support of engineering activities is the result of dynamic continuous development of the relevant virtual technology during the past two decades. More and more industrial products apply cyber decisions with real time physical execution. Any contribution such as OCM requires firm and reliable knowledge about actual modeling technology and platform at any participant. Availability of professionally proven engineering modeling platform is essential for all participants. Platform must be configured for all modeling and other capabilities which represent the required level. Any restriction of platform capabilities may result conservative and not actual content in OCM and can lead to malfunction even dangerous physical activity in smart CPS system.

DSAIAM decided establishing its virtual research laboratory (VRL) to serve research for OCM and other model centered studies by suitable virtual environment. DSAIAM joined to the 3DEXPERIENCE platform for Academia on the Cloud program of Dassault Systémes. This leading engineering modeling platform provides software capabilities in APPs for modeling (SaaS), means for customizing VRL for Ph.D. and other university research projects in platform (PaaS), and storage, virtual machine, language and other infrastructure (IaaS) services.

The main structure of VRL is introduced in Fig. 6. Platform services include platform management among others to grant roles for participants. VRL specific roles can be surveyed in the first column. Experimental model developer defines model system for research project. Researcher contextually places results and virtually executes them in experimental model system using an experiment plan. Research supervisor can follow research at any time. Industrial adviser brings valuable experience into the research. Modeling adviser gives assistance for researchers in model system related affairs. Finally, research process supervisor manages research project or a group of closely related research projects. Owing to the cloud environment, participants can access the VRL from anywhere and anytime in accordance with the authorization by the granted roles. Any participant has their own workspace.

Thematic sets of modeling capabilities (APPS) are available in modeling, simulation, information intelligence, and social and collaborative groups in the 3DEXPERIENCE. University and research specific functions and researchoriented modeling capabilities make it possible to configure the platform for the demands posed by specific tasks in VRL. Serving university research, VRL highly relies on fundamental, problem related, and experimental configuration related research capabilities in platform. More essential research purposed capabilities are demanded to provide integration with Mathlab, Simulink and Dymola systems and surfaces for Modelica and logic control modeler (LCM) languages in the platform. 


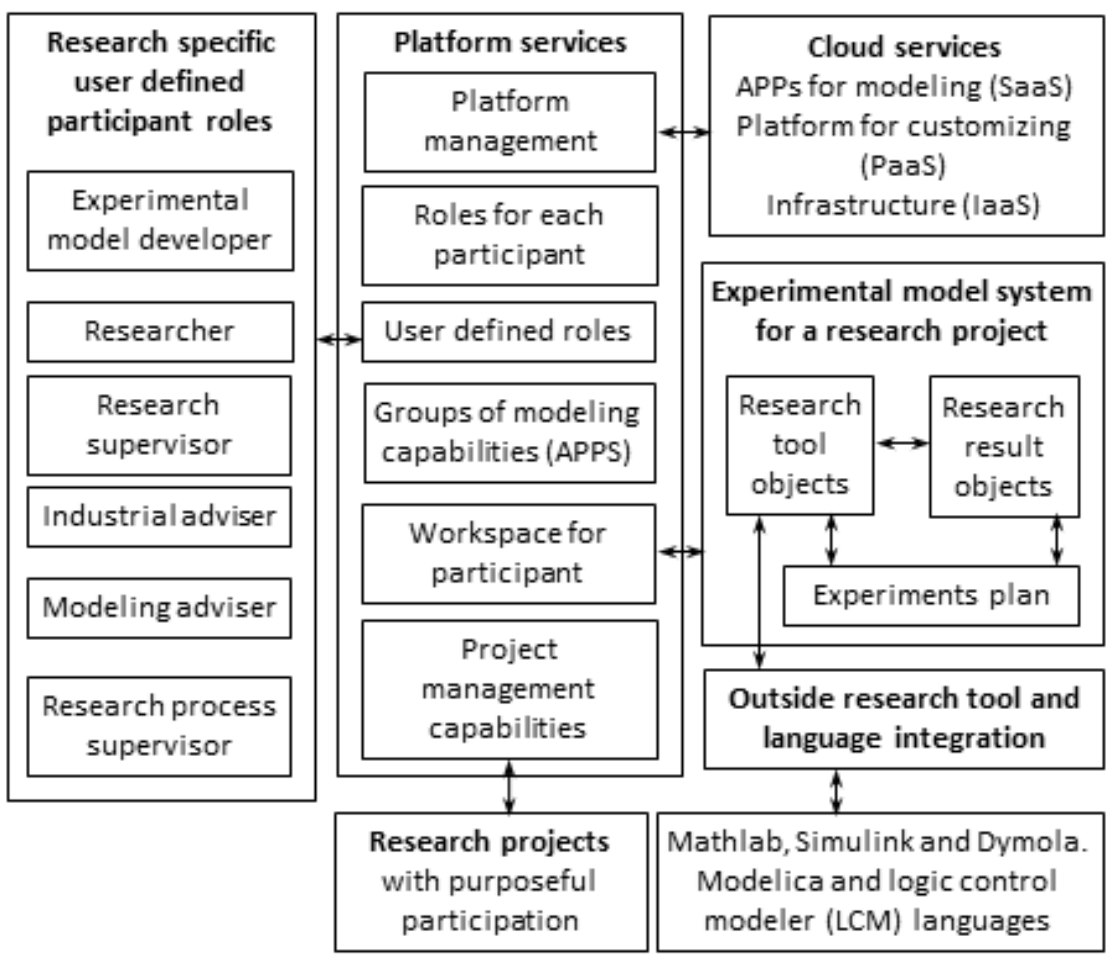

Figure 6

Virtual Research Laboratory (VRL)

As it was stated above, implementation of OCM related and other relevant university and Ph.D. project related research are planned for the VRL. These research programs demand wide area context representations in experimental model. Modeling environment, which is required to do this will be available in the VRL.

The next challenge is to configure experimental model in laboratory circumstances to include details from EPS enough for the experiments when the real world EPS may consist of hundreds of systems realized by a structure consisting of millions of mechanical, electrical, electronic, and software components. VRL is intended to be a flexible virtual research environment which allows wide variety of experimental configurations to develop and simulate. The selected platform provides research tools to integrated handling of theories, methodologies, practices, and experiences. This is a worldwide trend in most advanced model based engineering.

\section{Conclusions}

High level automation together with system structure, high complexity, and relying upon several disciplinaries as mathematics, physics, intelligent computing 
and systemics brought new era and new challenges in research for engineering. Latest integrated autonomous generic modeling and simulation have become essential at development and operation of advanced industrial and commercial products. In this scenario, product lifecycle developed consistent structure of driving contexts is essential glue in extended product system (EPS). Always actual structure of wide area contexts is firm requirement to assure renewable virtual and field operating smart CPS product.

Recognizing the above change in engineering, this paper introduces results of research in extended organized content model (OCM). OCM is suitable for representation of content behind driving contexts for an EPS in which driving contexts of object parameters establish active connection of OCM with PMS and cyber units of field operating smart CPS. Beyond role of filling the gap between outside contexts and EPS, the proposed OCM includes substructures to organize driving content for situation recognition related aspects. OCM is developed for lifecycle making continuous engineering (CE) possible with actual content and availability of content preliminaries. It must be emphasized that OCM needs continuous exceptional level work for life cycle of EPS. OCM is a specific organized storage of context positioned knowledge, which is used at EPS related engineering.

Experimental model centered VRL is under installation at the DSAIAM as virtual laboratory for realization research among other in OCM using Dassult Systémes's 3DEXPERIENCE engineering modeling platform on the cloud. DSAIAM intends to prepare itself to the future in this way.

\section{Acknowledgement}

The author gratefully acknowledges the financial support by the Óbuda University and the Doctoral School of Applied Informatics and Applied Mathematics.

\section{References}

[1] L. Horváth: In the Main Stream of Emerging Engineering, in proc of the $16^{\text {th }}$ IEEE International Symposium on Computational Intelligence and Informatics, Budapest, Hungary, 2015, pp. 11-20

[2] V. Gomez-Jauregui, F. Cue-Palencia, C. Manchado, C. Otero: Education for the Industry of the Future (IoF) with the 3D Experience Platform, in book Advances on Mechanics, Design Engineering and Manufacturing II, Springer, 2019, pp. 761-769

[3] L. Horváth: Virtual Research Laboratory for Smart Engineering in the Cloud, in proc. of the IEEE $13^{\text {th }}$ International Symposium on Applied Computational Intelligence and Informatics, Timişoara, Romania, 2019, pp. 179-184

[4] L. Horváth: New Method for Definition of Organized Driving Chains in Industrial Product Model, in Proc. of the 2017 IEEE International 
Conference on Industrial Technology, Toronto, Canada, 2017, pp. 11831188

[5] L. Horváth: Intellectual Content Driving for Model of Industrial Cyber Physical System, in Proc. of the 2019 IEEE International Conference on Systems, Man and Cybernetics, Bari, Italy, 2019, pp. 1394-1399

[6] B. Fitzgerald, K-J. Stol: Continuous software engineering: A roadmap and agenda, Journal of Systems and Software, Vol. 123, 2017, pp. 176-189

[7] K. Baughey: Functional and Logical Structures: A Systems Engineering Approach," SAE 2011 World Congress, SAE Technical Paper 2011-010517,2011

[8] S. Kleiner, C. Kramer: Model Based Design with Systems Engineering Based on RFLP Using V6, in Smart Product Engineering, Springer, 2013, pp. 93-102

[9] L. Horváth: Supporting Lifecycle Management of Product Data by Organized Descriptions and Behavior Definitions of Engineering Objects, In Journal of Advanced Computational and Intelligent Informatics, Tokyo, Vol. 11, No. 9, 2007, pp. 1-7

[10] R. L. Ackoff: From Data to Wisdom, in Journal of Applied Systems Analysis, Vol. 16, No. 1, 1989, pp. 3-9

[11] I. Rudas, L. Horváth: Modeling of Manufacturing Processes Using Petri Net Representation, Engineering Applications of Artificial Intelligence, Vol. 10, No. 3, 1997, pp. 243-255

[12] L. Horváth, I. Rudas: Intelligent Computer Methods for Modeling of Manufacturing Processes and Human Intent, Journal of Advanced Computational Intelligence and Intelligent Informatics, Vol. 2, No. 3, 1998, pp. 111-119

[13] L. Horváth, I. Rudas: Modeling of Manufacturing Processes in Simultaneous Engineering Using Collaborative Methods and Tools, In book Simultaneous Engineering Methodologies and Applications, Gordon and Breach Publisher, 1999, pp. 321-357

[14] L. Horváth, I. Rudas: Emerging Intelligent Technologies in Computer Aided Engineering, Journal of Advanced Computational Intelligence and Intelligent Informatics Vol. 4, No. 4, 2000, pp. 268-278

[15] L. Horváth, I. Rudas: Virtual technology based associative integration of modeling of mechanical parts, Journal of Advanced Computational Intelligence and Intelligent Informatics Vol. 5, No. 5, 2001, pp. 269-278

[16] L. Horváth, I. Rudas: Integrating expert knowledge in behavioral feature based product models, in Proc. of the $28^{\text {th }}$ Annual Conference of the IEEE Industrial Electronics Society, Sevilla, Spain, 2002, pp. 2479-2484 
[17] L. Horváth, I. Rudas: Modeling Behavior of Engineering Objects Using Design Intent Model, in Proc. of the $28^{\text {th }}$ Annual Conference of the IEEE Industrial Electronics Society, Roanoke, VA, USA, 2003, pp. 872-876

[18] L. Horváth, I. Rudas: Modeling and Problem Solving Techniques for Engineers, Elsevier Academic Press, 2004

[19] L. Horváth, I. Rudas: Human Intent Description in Environment Adaptive Product Model Objects, Journal of Advanced Computational Intelligence and Intelligent Informatics Vol. 9, No. 4, 2005, pp. 415-422

[20] L. Horváth, I. Rudas: Intelligence for Assistance of Engineering Decisions in Modeling Systems, Studies in Informatics and Control, Vol. 15, No. 3, 2006, pp. 297-306

[21] L. Horváth, I. Rudas: An approach to processing product changes during product model based engineering, IEEE International Conference on System of Systems Engineering, San Antonio, TX, USA, 2007, Paper No.: 4304267

[22] L. Horváth, I. Rudas: Towards the information content driven product model, IEEE International Conference on System of Systems Engineering, Monterey, CA, USA, 2008, Paper No.: 4724183

[23] L. Horváth, I. Rudas: Human Intent Representation in Knowledge Intensive Product Model, Journal of Computers, Vol. 4, No. 10, 2009, pp. 954-961

[24] L. Horváth, I. J. Rudas: Knowledge Technology for Product Modeling, in book Knowledge in Context-Few Faces of the Knowledge Society, Wolters Kluwer, 2010, pp. 113-137

[25] L. Horváth, I. J. Rudas: Integrated Engineering Processes in Virtual Product Definition, in proc of the IEEE International Conference on Systems, Man, and Cybernetics, Anchorage, AK, USA, 2011, pp. 2996-3001

[26] L. Horváth, I. J. Rudas: Adaptive Method for Knowledge Driven Definition of Products in Modeling Systems, in proc. of the World Automation Congress, Puerto Vallarta, Mexico, 2012, pp. 1-6

[27] L. Horváth, I. J. Rudas: Active Knowledge for the Situation-driven Control of Product Definition, Acta Polytechnica Hungarica, Vol. 10, No. 2, pp. 217-234 (2013)

[28] L. Horváth, I. J. Rudas: New Method for Generation of RFLP Structure Elements in PLM Model, in book New Trends in Software Methodologies, Tools and Techniques, IOS Press, 2014, pp. 310-324

[29] L. Horváth, I. J. Rudas: Product Behavior Definition for Element Generation in RFLP Structure, in book New Trends on System Sciences and Engineering, IOS Press, 2015, pp. 485-498 
[30] L. Horváth, I. J. Rudas: New Approach to Multidisciplinary Content Driving of Engineering Model System Component Generation, in book New Trends in Software Methodologies, Tools and Techniques, IOS Press, 2016, pp. 38-49

[31] L. Horváth, I. J. Rudas: Driving Engineering Model Generation on Functional and Logical Levels, in book New Trends in Software Methodologies, Tools and Techniques, IOS Press, 2017, pp. 678-690

[32] L. Horváth, I. J. Rudas: Information Content Driven Model for Virtual Engineering Space, Acta Polytechnica Hungarica, Vol. 15, No. 2, 2018, pp. 7-32

[33] L. Horváth: Smart Engineering Modeling for Smart Industrial Products, Acta Polytechnica Hungarica, Vol. 16, No. 10, 2019, pp. 11-30

[34] M. Abramovici, J. C. Göbel, M. Neges: Smart Engineering as Enabler for the $4^{\text {th }}$ Industrial Revolution, in book Integrated Systems: Innovations and Applications, Springer, 2015, pp. 163-170

[35] M. R. Endsley: Situation Awareness in Future Autonomous Vehicles: Beware of the Unexpected, in Proc. of the $20^{\text {th }}$ Congress of the International Ergonomics Association, Florence, Italy, 2018, pp 303-309

[36] P. Leitao, A. W. Colombo, S. Karnouskos: Industrial automation based on cyber-physical systems technologies: Prototype implementations and challenges, in Computers in Industry, Vol. 81, 2016, pp. 11-25

[37] J Lefèvre, S Charles, M Bosch-Mauchand, B Eynard, É Padiolleau: Multidisciplinary modelling and simulation for mechatronic design, Journal of Design Research, Vol. 12, No. 1-2, 2014, pp. 127-144

[38] A Canedo, E Schwarzenbach, E. M A Al Faruque: Context-sensitive synthesis of executable functional models of cyber-physical systems, In Proc. of the 2013 ACM/IEEE International Conference on Cyber-Physical Systems (ICCPS), Philadelphia, PA, USA, 2013, pp. 99-108 